\title{
Kernelized Probabilistic Matrix Factorization: Exploiting Graphs and Side Information
}

\author{
Tinghui Zhou* Hanhuai Shan* Arindam Banerjee* Guillermo Sapiro ${ }^{\dagger}$
}

\begin{abstract}
We propose a new matrix completion algorithmKernelized Probabilistic Matrix Factorization (KPMF), which effectively incorporates external side information into the matrix factorization process. Compared with Probabilistic Matrix Factorization (PMF) [1], which imposes a Gaussian prior for each row of the data matrix, KPMF imposes a Gaussian Process (GP) prior over all rows of the matrix, hence the learned model explicitly captures the underlying correlation among the rows (and the same holds for columns). This crucial difference greatly boosts the performance of KPMF when appropriate side information, e.g., users' social network in recommender systems, is incorporated. Furthermore, GP priors allow the KPMF model to fill in a row that is entirely missing in the original matrix based on the side information alone, which is not feasible for standard PMF formulation. In our paper, we mainly work on the matrix completion problem with a graph among the rows and/or columns as side information, but the proposed framework can be easily extended to handle other types of side information as well. Finally, we demonstrate the efficacy of KPMF through two different applications: 1) item recommendation and 2) image restoration.
\end{abstract}

\section{Introduction}

The problem of missing value prediction, and particularly matrix completion, has been addressed in many research areas, including recommender systems [2, 3], geostatistics [4], and image restoration [5]. In such problems, we are typically given an $N \times M$ data matrix $R$ with a number of missing entries, and the goal is to fill in the missing entries properly such that they are coherent with the existing data, where the existing data may include the observed entries in the data matrix as well as the side information depending on the specific problem domain.

Among the existing matrix completion techniques,

\footnotetext{
${ }^{*}$ Department of Computer Science and Engineering, University of Minnesota, Twin Cities

${ }^{\dagger}$ Department of Electrical and Computer Engineering, University of Minnesota, Twin Cities
}

factorization based algorithms have achieved great success and popularity $[1,6-8]$. In these algorithms, each row, as well as each column, of the matrix has a latent vector, obtained from factorizing the partially observed matrix. The prediction of each missing entry is thus the inner product of latent vectors of the corresponding row and the corresponding column. However, most such techniques suffer from the data sparsity problem, which unfortunately, is often encountered in real-world scenarios. For instance, according to [2], the density of non-missing ratings in most commercial recommender systems is less than $1 \%$. It is thus very difficult to do missing value prediction based on such small amount of data. On the other hand, in addition to the data matrix, other sources of information, e.g., users' social network in recommender systems, are sometimes readily available, and could provide key information about the underlying model. However, many of the existing factorization techniques simply ignore such side information, or intrinsically, are not capable of exploiting it (see Section 2 for more on related work)

To overcome such limitations, we propose the Kernelized Probabilistic Matrix Factorization (KPMF) model, which incorporates the side information through kernel matrices over rows and over columns. In essence, KPMF models a matrix as the product of two latent matrices, which are sampled from two different zero-mean Gaussian processes (GP). The covariance functions of the GPs are derived from the side information, and encode the correlational structure of rows and columns respectively. In this paper, we focus on deriving covariance functions from undirected graphs (e.g. users' social network). However, the general framework of our algorithm allows it to incorporate other types of side information as well. For instance, when the side information is in the form of feature vectors, we may use the RBF kernel [14] as the covariance function.

Although KPMF seems highly related to the Probabilistic Matrix Factorization (PMF) [1] and its generalized counterpart - Bayesian PMF (BPMF) [7], the key difference that makes KPMF a more powerful and more representative model is as follows: while PMF and BPMF put a Gaussian prior on the latent vector 
of each row, KPMF puts a Gaussian process prior over all rows. Therefore, KPMF is able to capture the correlations among the rows and/or columns, while such correlations are entirely lost in PMF and BPMF. Moreover, PMF and BPMF need at least one observed entry in a row to predict the rest. When an entire row is missing, they fail to make prediction for that row. In contrast, KPMF could still make prediction based on the row correlations alone.

We demonstrate KPMF through two applications: 1) item recommendation and 2) image restoration. For item recommendation, we have a partially observed rating matrix from users to items, and the side information is users' social network. For image restoration, the image itself is a matrix, and the corrupted regions are what to be predicted. We construct a graph both for the rows and for the columns of the image based on the spatial smoothness assumption, and utilize them as the side information. Our experiments show that KPMF consistently outperforms state-of-the-art collaborative filtering algorithms, and also generate satisfactory results for image restoration.

The rest of the paper is organized as follows: Section 2 discusses related work. Section 3 gives a brief overview of the background, and in particular, the PMF and BPMF models. Section 4 presents the KPMF model and two methods, gradient descent and stochastic gradient descent, for learning it. We present the experimental results for recommender systems in Section 5 and for image restoration in Section 6, and conclude in Section 7.

\section{Related Work}

Factorization based algorithms are very powerful techniques for matrix completion. In particular, recently, a probabilistic framework for matrix factorization, namely Probabilistic Matrix Factorization (PMF), is proposed in [1], and generalized to a full Bayesian model in [7] (see Section 3.2 for more discussion on PMF and Bayesian PMF). Additionally, [6] develops a non-linear extension to PMF using Gaussian process latent variable models. However, one major flaw of the methods above is the lack of ability to incorporate side information into the factorization process.

Now we briefly review some prior work on matrix completion with the aid of side information. For collaborative filtering, the approach proposed in [8] generalizes PMF to a parametric framework and uses topic models for incorporating side information, while [3] performs probabilistic matrix factorization on both users' social network and the rating matrix jointly, so that the resulting latent matrices depend on both input sources. Another approach relevant to our work is Linear Models of Coregionalization (LMC). LMC is a family of spatial process models widely used in multivariate geostatistics [4]. In LMCs, the data matrix is first transformed to a vectorized form, and then the covariance structures across the original rows and columns are captured in this long vector. However, scalability becomes an issue for LMC when the data size becomes large. In [9], the authors propose the Probabilistic Matrix Addition (PMA) model, where the generative process of the data matrix is modeled as the additive combination of latent matrices drawn from two Gaussian processes: one for the rows, and the other for the columns. The main difference between PMA and KPMF is that in PMA, the Gaussian processes capturing the covariance structure for rows and columns are combined additively in the generative model, while in KPMF, the Gaussian processes are priors for the row and column latent matrices, and the data matrix is generated from the product of the two latent matrices.

\section{Preliminaries}

3.1 Notations While the proposed KPMF model is applicable to other matrix completion problems, we focus on the collaborative filtering application and develop the notation and exposition accordingly. We define the main notations used in this paper as follows:

$R-N \times M$ data matrix.

$R_{n,:}-n^{\text {th }}$ row of $R$.

$R_{:, m}-m^{t h}$ column of $R$.

$N$ - Number of rows in $R$.

$M$ - Number of columns in $R$.

$D$ - Dimension of the latent factors.

$U-N \times D$ latent matrix for rows of $R$.

$V-M \times D$ latent matrix for columns of $R$.

$U_{n,:} \in \mathbb{R}^{D}$ - Latent factors for $R_{n,:}$.

$V_{m,:} \in \mathbb{R}^{D}$ - Latent factors for $R_{:, m}$.

$U_{:, d} \in \mathbb{R}^{N}-d^{\text {th }}$ latent factor for all rows of $R$.

$V_{:, d} \in \mathbb{R}^{M}-d^{t h}$ latent factor for all columns of $R$.

$K_{U} \in \mathbb{R}^{N \times N}$ - Covariance matrix for rows.

$K_{V} \in \mathbb{R}^{M \times M}$ - Covariance matrix for columns.

$S_{U} \in \mathbb{R}^{N \times N}$ - Inverse of $K_{U}$.

$S_{V} \in \mathbb{R}^{M \times M}-$ Inverse of $K_{V}$.

$[n]_{1}^{N}-n=\{1,2, \ldots, N\}$.

3.2 PMF and BPMF Consider an $N \times M$ realvalued matrix $R$ with a number of missing entries. The goal of matrix completion is to predict the values of those missing entries. Probabilistic Matrix Factorization (PMF) [1] approaches this problem from the matrix factorization aspect. Assuming two latent matrices: $U_{N \times D}$ and $V_{M \times D}$, with $U$ and $V$ capturing the 


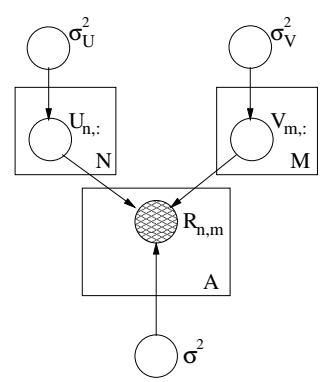

(a)

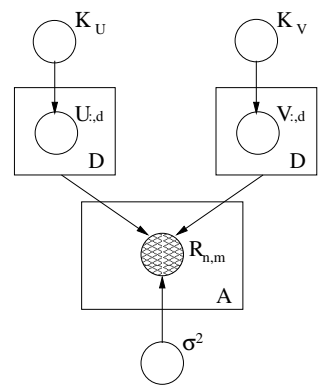

(b)
Figure 1: (a) The generative process of $R$ in PMF; (b) The generative process of $R$ in KPMF. $A$ is the total number of non-missing entries in the matrix.

row and the column features of $R$ respectively, the generative process for PMF is given as follows (also see Figure 1 (a)):

1. For each row $n$ in $R,[n]_{1}^{N}$, generate $U_{n,:} \sim$ $\mathcal{N}\left(\mathbf{0}, \sigma_{U}^{2} I\right)$, where $I$ denotes the identity matrix.

2. For each column $m$ in $R,[m]_{1}^{M}$, generate $V_{m,:} \sim$ $\mathcal{N}\left(\mathbf{0}, \sigma_{V}^{2} I\right)$.

3 . For each of the non-missing entries $(n, m)$, generate $R_{n, m} \sim \mathcal{N}\left(U_{n,:} V_{m,:}^{T}, \sigma^{2}\right)$.

The model has zero-mean spherical Gaussian priors on $U_{n,:}$ and $V_{m,:}$, and each entry $R_{n, m}$ is generated from a univariate Gaussian with the mean determined by the inner product of $U_{n,:}$ and $V_{m,:}$. The log-posterior over the latent matrices $U$ and $V$ is given by:

$$
\begin{aligned}
& \log p\left(U, V \mid R, \sigma^{2}, \sigma_{U}^{2}, \sigma_{V}^{2}\right) \\
= & -\frac{1}{2 \sigma^{2}} \sum_{n=1}^{N} \sum_{m=1}^{M} \delta_{n, m}\left(R_{n, m}-U_{n,:} V_{m,:}^{T}\right)^{2} \\
& -\frac{1}{2 \sigma_{U}^{2}} \sum_{n=1}^{N} U_{n,:}^{T} U_{n,:}-\frac{1}{2 \sigma_{V}^{2}} \sum_{m=1}^{M} V_{m,:}^{T} V_{m,:} \\
& -\frac{1}{2}\left(A \log \sigma^{2}+N D \log \sigma_{U}^{2}+M D \log \sigma_{V}^{2}\right)+C,
\end{aligned}
$$

where $\delta_{n, m}$ is the indicator taking value 1 if $R_{n, m}$ is an observed entry, and 0 otherwise, $A$ is the number of non-missing entries in $R$, and $C$ is a constant that does not depend on the latent matrices $U$ and $V$. MAP inference maximizes the log-likelihood with respect to $U$ and $V$, which could then be used to predict the missing entries in $R$.

As an extension of PMF, Bayesian PMF (BPMF) [7] introduces a full Bayesian prior for each $U_{n, \text { : }}$ and each $V_{m,:} U_{n,:}$ (and similarly for $V_{m,:}$ ) is then sampled from $\mathcal{N}\left(\mu_{U}, \Sigma_{U}\right)$, where the hyperparameters $\left\{\mu_{U}, \Sigma_{U}\right\}$ are further sampled from Gaussian-Wishart priors.

\section{KPMF}

In this section, we describe the proposed Kernelized Probabilistic Matrix Factorization (KPMF) model, and present both gradient descent and stochastic gradient descent methods for learning the model.

4.1 The Model In KPMF, the prior distribution of each column of the latent matrices, $U_{:, d}$ and $V_{:, d}$, is a zero-mean Gaussian process [10]. Gaussian processes are a generalization of the multivariate Gaussian distribution. While a multivariate Gaussian is determined by a mean vector and a covariance matrix, the Gaussian process $G P\left(m(x), k\left(x, x^{\prime}\right)\right)$ is determined by a mean function $m(x)$ and a covariance function $k\left(x, x^{\prime}\right)$. In our problem, $x$ is an index of matrix rows or columns. Without loss of generality, let $m(x)=\mathbf{0}$, and $k\left(x, x^{\prime}\right)$ denote the corresponding kernel function, which specifies the covariance between any pair of rows or columns. Also, let $K_{U} \in \mathbb{R}^{N \times N}$ and $K_{V} \in \mathbb{R}^{M \times M}$ denote the full covariance matrix for rows of $R$ and columns of $R$ respectively. As we shall see later, using $K_{U}$ and $K_{V}$ in the priors forces the factorization to capture the underlying correlations among rows and among columns simultaneously.

Assuming $K_{U}$ and $K_{V}$ are known, ${ }^{1}$ the generative process for KPMF is given as follows (also see Figure 1(b)):

1. Generate $U_{:, d} \sim G P\left(\mathbf{0}, K_{U}\right),[d]_{1}^{D}$.

2. Generate $V_{:, d} \sim G P\left(\mathbf{0}, K_{V}\right),[d]_{1}^{D}$.

3. For each non-missing entry $R_{n, m}$, generate $R_{n, m} \sim$ $\mathcal{N}\left(U_{n,:} V_{m,:}^{T}, \sigma^{2}\right)$, where $\sigma$ is a constant.

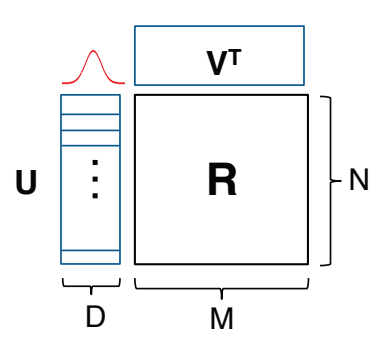

(a) PMF and BPMF

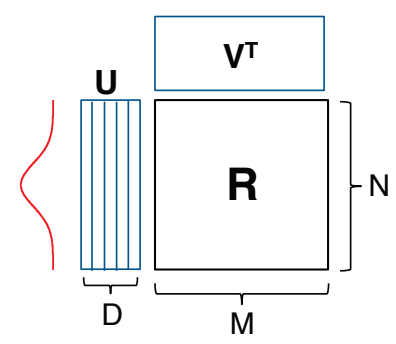

(b) KPMF
Figure 2: (a) $U$ is sampled in a "row-wise" manner in PMF and BPMF; (b) $U$ is sample in a "column-wise" manner in KPMF.

\footnotetext{
${ }^{1}$ The choice of $K_{U}$ and $K_{V}$ depends on the specific problem domain, and will be addressed later with particular examples.
} 
The likelihood over the observed entries in the target matrix $R$ given latent matrices $U$ and $V$ is

$$
p\left(R \mid U, V, \sigma^{2}\right)=\prod_{n=1}^{N} \prod_{m=1}^{M}\left[\mathcal{N}\left(R_{n, m} \mid U_{n,:} V_{m,:}^{T}, \sigma^{2}\right)\right]^{\delta_{n, m}},
$$

with the priors over $U$ and $V$ given by

$$
\begin{aligned}
& p\left(U \mid K_{U}\right)=\prod_{d=1}^{D} G P\left(U_{:, d} \mid 0, K_{U}\right), \\
& p\left(V \mid K_{V}\right)=\prod_{d=1}^{D} G P\left(V_{:, d} \mid 0, K_{V}\right) .
\end{aligned}
$$

For simplicity, we denote $K_{U}^{-1}$ by $S_{U}$, and $K_{V}^{-1}$ by $S_{V}$. The log-posterior over $U$ and $V$ is hence given by

$$
\begin{aligned}
& \log p\left(U, V \mid R, \sigma^{2}, K_{U}, K_{V}\right) \\
= & -\frac{1}{2 \sigma^{2}} \sum_{n=1}^{N} \sum_{m=1}^{M} \delta_{n, m}\left(R_{n, m}-U_{n,:} V_{m,:}^{T}\right)^{2} \\
& -\frac{1}{2} \sum_{d=1}^{D} U_{:, d}^{T} S_{U} U_{:, d}-\frac{1}{2} \sum_{d=1}^{D} V_{:, d}^{T} S_{V} V_{:, d} \\
& -A \log \sigma^{2}-\frac{D}{2}\left(\log \left|K_{U}\right|+\log \left|K_{V}\right|\right)+C,
\end{aligned}
$$

PMF is a simple special case of both models, with neither correlations across the rows, nor correlations across latent factors captured.

The row and column independencies in PMF and BPMF significantly undermine the power of the model, since strong correlations among rows and/or among columns are often present in real scenarios. For instance, in recommender systems, users' decisions on item ratings (represented by rows) are very likely to be influenced by other users who have social connections (friends, families, etc.) with them. PMF and BPMF fail to capture such correlational dependencies. As a result, the proposed KPMF performs considerably better than PMF and BPMF (see Section 5).

4.3 Gradient Descent for KPMF We perform a MAP estimate to learn the latent matrices $U$ and $V$, which maximize the log-posterior in (4.5), and is equivalent to minimizing the following objective function:

$$
\begin{aligned}
E= & \frac{1}{2 \sigma^{2}} \sum_{n=1}^{N} \sum_{m=1}^{M} \delta_{n, m}\left(R_{n, m}-U_{n,:} V_{m,:}^{T}\right)^{2} \\
& +\frac{1}{2} \sum_{d=1}^{D} U_{:, d}^{T} S_{U} U_{:, d}+\frac{1}{2} \sum_{d=1}^{D} V_{:, d}^{T} S_{V} V_{:, d} .
\end{aligned}
$$

Minimization of $E$ can be done through gradient descent. In particular, the gradients are given by

where $A$ is the total number of non-missing entries in $R$, $|K|$ is the determinant of $K$, and $C$ is a constant term not depending on the latent matrices $U$ and $V$.

4.2 KPMF Versus PMF/BPMF We illustrate the difference between $\mathrm{KPMF}$ and $\mathrm{PMF} / \mathrm{BPMF}$ in Figure 2. In PMF/BPMF, $U$ is sampled in a "row-wise" manner (Figure $2(\mathrm{a})$ ), i.e., $U_{n, \text { : }}$ is sampled for each row in $R$. $\left\{U_{n,:},[n]_{1}^{N}\right\}$ are hence conditionally independent given the prior. As a result, correlations among rows are not captured in the model. In contrast, in $\mathrm{KPMF}, U$ is sampled in a "column-wise" manner (Figure 2(b)), i.e., for each of the $D$ latent factors, $U_{:, d} \in \mathbb{R}^{N}$ is sampled for all rows of $R$. In particular, $U_{:, d}$ is sampled from a Gaussian process whose covariance $K_{U}$ captures the row correlations. In this way, during training, the latent factors of each row $\left(U_{n,:}\right)$ are correlated with those of all the other rows $\left(U_{n^{\prime} \text {,: }}\right.$ for $\left.n^{\prime} \neq n\right)$ through $K_{U}$. Roughly speaking, if two rows share some similarity according to the side information, the corresponding latent factors would also be similar after training, which is intuitively what we want to achieve given the side information. Similarly, the discussion above also applies to $V$ and the columns of $R$. The difference between KPMF and BPMF is subtle but they are entirely different models, and cannot be viewed as special cases of each other.

$$
\begin{aligned}
\frac{\partial E}{\partial U_{n, d}}= & -\frac{1}{\sigma^{2}} \sum_{m=1}^{M} \delta_{n, m}\left(R_{n, m}-U_{n,:} V_{m,:}^{T}\right) V_{d, m} \\
& +\boldsymbol{e}_{(n)}^{T} S_{U} U_{:, d} \\
\frac{\partial E}{\partial V_{m, d}}= & -\frac{1}{\sigma^{2}} \sum_{n=1}^{N} \delta_{n, m}\left(R_{n, m}-U_{n,:} V_{m,:}^{T}\right) U_{d, n} \\
& +\boldsymbol{e}_{(m)}^{T} S_{V} V_{:, d},
\end{aligned}
$$

where $\boldsymbol{e}_{(n)}$ denotes an $N$-dimensional unit vector with the $n^{\text {th }}$ component being one and others being zero. The update equations for $U$ and $V$ are

$$
\begin{aligned}
& U_{n, d}^{(t+1)}=U_{n, d}^{(t)}-\eta \frac{\partial E}{\partial U_{n, d}}, \\
& V_{m, d}^{(t+1)}=V_{m, d}^{(t)}-\eta \frac{\partial E}{\partial V_{m, d}},
\end{aligned}
$$

where $\eta$ is the learning rate. The algorithm updates $U$ and $V$ following (4.9) and (4.10) alternatively until convergence. It should be noted that since $K_{U}$ and $K_{V}$ remain fixed throughout all iterations, $S_{U}$ and $S_{V}$ need to be computed only once at initialization.

Now Suppose an entire row or column of $R$ is missing. While PMF and BPMF fail to address such problem, KPMF still works if appropriate side information 
is given. In this case, the update equations in (4.9) and (4.10) become

$$
\begin{aligned}
U_{n, d}^{(t+1)} & =U_{n, d}^{(t)}-\eta \boldsymbol{e}_{(n)}^{T} S_{U} U_{:, d} \\
& =U_{n, d}^{(t)}-\eta \sum_{n^{\prime}=1}^{N} S_{U}\left(n, n^{\prime}\right) U_{n^{\prime}, d} \\
V_{m, d}^{(t+1)} & =V_{m, d}^{(t)}-\eta \boldsymbol{e}_{(m)}^{T} S_{V} V_{:, d} \\
& =V_{m, d}^{(t)}-\eta \sum_{m^{\prime}=1}^{M} S_{V}\left(m, m^{\prime}\right) V_{m^{\prime}, d} .
\end{aligned}
$$

In this case, update of the corresponding $U_{n,:}$ is based on the weighted average of the current $U$ over all rows, including the rows that are entirely missing and the rows that are not, and the weights $S_{U}\left(n, n^{\prime}\right)$ reflect the correlation between the current row $n$ and the rest. The same holds for $V$ and the columns.

\subsection{Stochastic Gradient Descent for KPMF As} stochastic gradient descent (SGD) usually converges much faster than gradient descent, we also derive the SGD update equations for KPMF below.

The objective function in (4.6) could be rewritten as

$$
\begin{aligned}
E= & \frac{1}{\sigma^{2}} \sum_{n=1}^{N} \sum_{m=1}^{M} \delta_{n, m}\left(R_{n, m}-U_{n,:} V_{m,:}^{T}\right)^{2} \\
& +\operatorname{Tr}\left(U^{T} S_{U} U\right)+\operatorname{Tr}\left(V^{T} S_{V} V\right),
\end{aligned}
$$

where $\operatorname{Tr}(X)$ denotes the trace of matrix $X$. Moreover,

$$
\begin{aligned}
& \operatorname{Tr}\left(U^{T} S_{U} U\right)=\operatorname{Tr}\left(U U^{T} S_{U}\right) \\
= & \operatorname{Tr}\left\{\left(\begin{array}{cccc}
U_{1::}^{T} U_{1,:} & U_{1::}^{T} U_{2,:} & \ldots & U_{1,:}^{T} U_{N,:} \\
U_{2,:}^{T} U_{1,:} & U_{2,:}^{T} U_{2,:} & \ldots & U_{2,:}^{T} U_{N,:} \\
\vdots & \vdots & \ddots & \vdots \\
U_{N,:}^{T} U_{1,:} & U_{N,:}^{T} U_{2,:} & \ldots & U_{N,:}^{T} U_{N,:}
\end{array}\right)\right. \\
& \left.\left(\begin{array}{cccc}
S_{U}(1,1) & S_{U}(1,2) & \ldots & S_{U}(1, N) \\
S_{U}(2,1) & S_{U}(2,2) & \ldots & S_{U}(2, N) \\
\vdots & \vdots & \ddots & \vdots \\
S_{U}(N, 1) & S_{U}(N, 2) & \ldots & S_{U}(N, N)
\end{array}\right)\right\} \\
= & \sum_{n=1}^{N} \sum_{n^{\prime}=1}^{N} S_{U}\left(n, n^{\prime}\right) U_{n,:}^{T} U_{n^{\prime},:},
\end{aligned}
$$

and similarly,

$$
\operatorname{Tr}\left(V^{T} S_{V} V\right)=\sum_{m=1}^{M} \sum_{m^{\prime}=1}^{M} S_{V}\left(m, m^{\prime}\right) V_{m,:}^{T} V_{m,:} .
$$

Therefore, (4.13) becomes

$E=\sum_{n=1}^{N} \sum_{m=1}^{M} \delta_{n, m}\left[\frac{1}{\sigma^{2}}\left(R_{n, m}-U_{n,:} V_{m,:}^{T}\right)^{2}\right.$

$$
\begin{aligned}
& +\frac{1}{\tilde{M}_{n}} U_{n,:}^{T} \sum_{n^{\prime}=1}^{N} S_{U}\left(n, n^{\prime}\right) U_{n^{\prime},:} \\
& \left.+\frac{1}{\tilde{N}_{m}} V_{m,:}^{T} \sum_{m^{\prime}=1}^{M} S_{V}\left(m, m^{\prime}\right) V_{m^{\prime},:}\right]=\sum_{n=1}^{N} \sum_{m=1}^{M} \delta_{n, m} E_{n, m},
\end{aligned}
$$

where $\tilde{M}_{n}$ is the number of non-missing entries in row $n$ and $\tilde{N}_{m}$ is the number of non-missing entries in column $m$. Finally, for each non-missing entry $(n, m)$, taking gradient of $E_{n, m}$ with respect to $U_{n,:}$ and $V_{m,:}$ gives:

$$
\begin{aligned}
\frac{\partial E_{n, m}}{\partial U_{n,:}} & =-\frac{2}{\sigma^{2}}\left(R_{n, m}-U_{n,:}^{T} V_{m,:}\right) V_{m,:} \\
& +\frac{1}{\tilde{M}_{n}}\left[\sum_{n^{\prime}=1}^{N} S_{U}\left(n, n^{\prime}\right) U_{n^{\prime},:}+S_{U}(n, n) U_{n,:}\right] \\
\frac{\partial E_{n, m}}{\partial V_{m,:}} & =-\frac{2}{\sigma^{2}} \delta_{n, m}\left(R_{n, m}-U_{n,:}^{T} V_{m,:}\right) U_{n,:} \\
& +\frac{1}{\tilde{N}_{m}}\left[\sum_{m^{\prime}=1}^{M} S_{V}\left(m, m^{\prime}\right) V_{m^{\prime},:}+S_{V}(m, m) V_{m,:}\right] .
\end{aligned}
$$

4.5 Learning KPMF with Diagonal Kernels If both $K_{U}$ and $K_{V}$ are diagonal matrices, meaning that both the rows and the columns are drawn i.i.d., KPMF reduces to PMF. If only one of the kernels is diagonal, it turns out that the corresponding latent matrix can be marginalized, yielding a MAP inference problem on one latent matrix. To illustrate this, suppose $K_{V}$ is diagonal $\left(K_{V}=\sigma_{V}^{2} I\right)$. Following the derivation in [6], the likelihood for each column in $R$ is given by

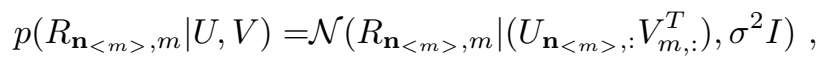

where $\mathbf{n}_{<m>}$ denotes the row indices of non-missing entries in the $m^{t h}$ column, so if there are $\tilde{N}$ non-missing entries in the column $m, R_{\mathbf{n}_{<m>, m}}$ is a $\tilde{N}$-dimensional vector, and $U_{\mathbf{n}_{<m>},}$ is a $\tilde{N} \times D$ matrix with each row corresponding to a non-missing entry in column $m$.

Since $p\left(V_{m,:} \mid \sigma_{V}^{2}\right)=\mathcal{N}\left(V_{m,:} \mid 0, \sigma_{V}^{2} I\right)$, we can marginalize over $V$, obtaining

$$
\begin{aligned}
& p(R \mid U)=\prod_{m=1}^{M} p\left(R_{\mathbf{n}_{<m>}, m} \mid U\right) \\
& =\prod_{m=1}^{M} \int_{V_{m,:}} \mathcal{N}\left(R_{\mathbf{n}_{<m>, m}} \mid\left(U_{\mathbf{n}_{<m>,:}} V_{m,:}^{T}\right), \sigma^{2} I\right) \\
& \times \mathcal{N}\left(V_{m,:} \mid 0, \sigma_{V}^{2} I\right) d V_{m,:} \\
& =\prod_{m=1}^{M} \mathcal{N}\left(0, \quad \sigma_{V}^{2} U_{\mathbf{n}_{<m>,}:} U_{\mathbf{n}_{<m>,}^{T}}+\sigma^{2} I\right)
\end{aligned}
$$


The objective function then becomes:

$$
\begin{aligned}
E= & \sum_{m=1}^{M}\left(R_{\mathbf{n}_{<m>}, m}^{T} C^{-1} R_{\mathbf{n}_{<m>, m}}+\log |C|\right) \\
& +\sum_{d=1}^{D} U_{:, d}^{T} S_{U} U_{:, d}
\end{aligned}
$$

where $C=\sigma_{V}^{2} U_{\mathbf{n}_{<m>,}:} U_{\mathbf{n}_{<m>,}^{T}}^{T}+\sigma^{2} I$. Moreover, since $V$ is no longer a term in the objective function, the gradient descent could be performed solely on $U$. However, in this case, updating $U$ at each iteration involves the inversion of $C$, which becomes computationally prohibitive when $N$ is large. Hence we do not use this formulation in our experiments.

4.6 Prediction Learning based on gradient descent or SGD gives us the estimate of the latent matrices $\hat{U}$ and $\hat{V}$. For any missing entry $R_{n, m}$, the maximumlikelihood estimation is the inner product of the corresponding latent vectors, i.e., $\hat{R}_{n, m}=\hat{U}_{n,:} \hat{V}_{m,:}^{T}$.

\section{Experiments on Recommender Systems}

In this section, we evaluate the KPMF model for item recommendation with known user relations. In particular, we are given a user-item rating matrix with missing entries as well as a social network graph among users (see Figure 3). The goal is to predict the missing entries in the rating matrix by exploiting both the observed ratings and the underlying rating constraints derived from the social network. We run experiments on two publicly available datasets, i.e. Flixster [11] and Epinion [12], and compare the prediction results of KPMF with several other algorithms.

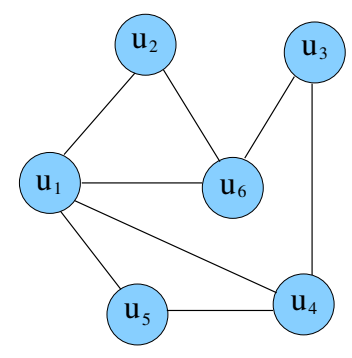

(a) Social network graph

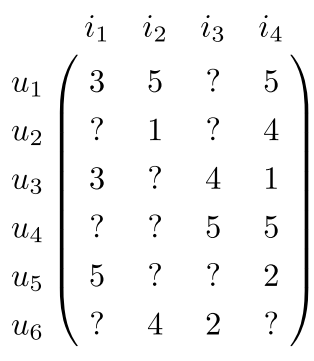

(b) Rating matrix
Figure 3: Example input data: (a) social network among 6 users; (b) observed rating matrix for the 6 users on 4 items.

5.1 Datasets Flixster $^{2}$ is a social movie website, where users can rate movies and make friends at the

\begin{tabular}{|l|c|c|}
\hline & Flixster & Epinion \\
\hline \hline \# Users & 2000 & 2000 \\
\hline \# Items & 3000 & 3000 \\
\hline \# Ratings & 173,172 & 60,485 \\
\hline \# Relations & 32,548 & 74,575 \\
\hline Rating Density & $2.89 \%$ & $1.00 \%$ \\
\hline
\end{tabular}

Table 1: Statistics of the datasets used.

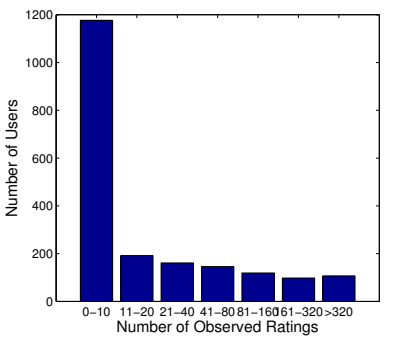

(a) Flixster

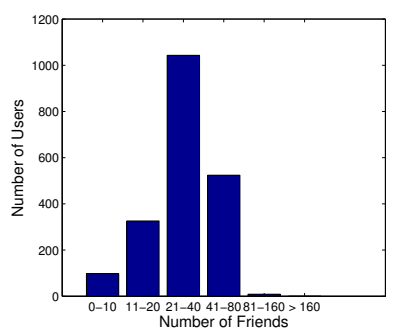

(c) Flixster

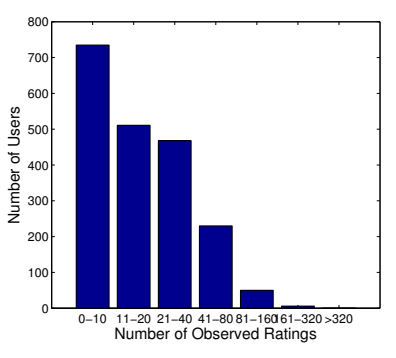

(b) Epinion

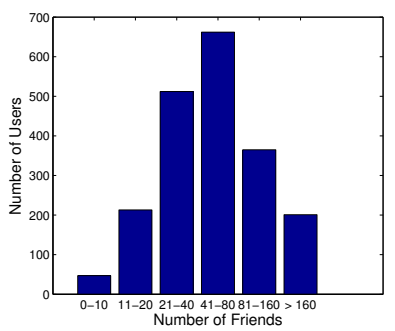

(d) Epinion
Figure 4: (a) and (b): Histograms of users' rating frequencies. (c) and (d): Histograms of the number of friends for each user.

same time. The social graph in Flixster is undirected, and the rating values are 10 discrete numbers ranging from 0.5 to 5 in steps of 0.5 .

Epinion $^{3}$ is a customer review website where users share opinions on various types of items such as electronic products, companies, and movies, through writing reviews or giving ratings. Each user also maintains a list of people he/she trusts, which forms a social network with trust relationships. Unlike Flixster, social network in Epinion is an directed graph, but for simplicity, we convert the directed edges to be undirected ones by keeping only one edge between two users if they are connected in either way originally. The rating values in Epinion are discrete values ranging from 1 to 5 .

For each dataset, we sampled a subset with 2,000 users and 3,000 items. For the purpose of testing our hypothesis - whether the social network could help in making ratings prediction-the 2,000 users selected are users with most friends in the social network, while the 3,000 items selected are the most frequently rated

\footnotetext{
${ }^{3} \mathrm{www}$.epinions.com
} 
overall. The statistics of the datasets are given in Table 1. Figure 4 shows the histograms for the number of past ratings and number of friends each user has.

Unless otherwise specified, from all the ratings, we randomly hold out $10 \%$ ratings as the validation set and another $10 \%$ as the test set. The rest $80 \%$ of ratings, along with the whole social network, are used for creating the training sets. To better evaluate the effect of social network, we have 4 training sets that are increasingly sparse, i.e., we use not only all the $80 \%$ but also $60 \%, 40 \%$, and $20 \%$ of the ratings to create 4 different training sets (note that the social network remains the same). For each observed rating $r$, we normalize it to $[0.2,1]$ using $r / r_{\max }$, where $r_{\max }$ is the maximum possible value for the ratings.

5.2 Graph Kernels To construct kernel matrices suitable to our problem, we consider the users' social network as an undirected, unweighted graph $G$ with nodes and edges representing users and their connections. Elements in the adjacency matrix of $G$ are determined by $A_{i, j}=1$ if there's an edge between user $i$ and $j$, and 0 otherwise. The Laplacian matrix [13] of $G$ is defined as $L=D-A$, where the degree matrix $D$ is a diagonal matrix with diagonal entries $d_{i}=\sum_{j=1}^{N} A_{i, j}(i=1, \ldots, N)$.

Graph kernels provide a way of capturing the intricate structure among nodes in a graph (If instead we are given features or attributes of the users, we could replace graph kernels with polynomial kernels, RBF kernels, etc. [14]). In our case, a graph kernel defines a similarity measure for users' taste on certain items. Generally speaking, users tend to have similar taste with their friends and families, and thus their ratings for the same items would also be correlated. Graph kernels could capture such effects in the social network, and the resulted kernel matrix would provide key information about users' rating patterns.

In this work, we examine three different graph kernels and refer readers to [15] for more available choices.

Diffusion kernel: The Diffusion kernel proposed in [15] is derived from the idea of matrix exponential, and has a nice interpretation on the diffusion process of substance such as heat. In particular, if we let some substance be injected at node $i$ and flow along the edges of the graph, $K_{D}(i, j)$ can be regarded as the amount of the substance accumulated at node $j$ in the steady state. The diffusion kernel intuitively captures the global structure among nodes in the graph, and it can be computed as follows:

$$
K_{D}=\lim _{n \rightarrow \infty}\left(1-\frac{\beta L}{n}\right)^{n}=e^{-\beta L},
$$

where $\beta$ is the bandwidth parameter that determines the extent of diffusion ( $\beta=0$ means no diffusion).

Commute Time (CT) kernel: As proposed in [16], the Commute Time kernel is closely related to the socalled average commute time (the number of steps a random walker takes to commute between two nodes in a graph), and can be computed using the pseudo-inverse of the Laplacian matrix: $K_{C T}=L^{\dagger}$.

Moreover, since $K_{C T}$ is conditionally positive definite, $\sqrt{K_{C T}(i, j)}$ behaves exactly like an Euclidean distance between nodes in the graph [17]. As a consequence, the nodes can be isometrically embedded in the subspace of $\mathbb{R}^{n}$ ( $n$ is the number of nodes), where the Euclidean distance between the points is $\sqrt{K_{C T}(i, j)}$.

Regularized Laplacian (RL) kernel: Smola and Kondor [18] introduce a way of performing regularization on graphs that penalizes the variation between adjacent nodes. In particular, it turns out that the graph Laplacian could be equally defined as a linear operator on the nodes of the graph, and naturally induces a seminorm on $\mathbb{R}^{n}$. This semi-norm quantifies the variation of adjacent nodes, and could be used for designing regularization operators. Furthermore, such regularization operators give rise to a set of graph kernels, and among them is the Regularized Laplacian kernel:

$$
K_{R L}=(I+\gamma L)^{-1},
$$

where $\gamma>0$ is a constant.

5.3 Methodology Given the social network, we use the Diffusion kernel, the CT kernel, and the RL kernel described above to generate the covariance matrix $K_{U}$ for users. The parameter setting is as follows: $\beta=$ 0.01 for the Diffusion kernel and $\gamma=0.1$ for the RL kernel, which are chosen via validation. Since no side information is available for the items in the Flixster or Epinion dataset, $K_{V}$ is assumed diagonal: $K_{V}=\sigma_{V}^{2} I$, where $I$ is an $M \times M$ identity matrix.

Given the covariance matrix $K_{U}$ generated from the graph kernels, we perform gradient descent (KPMF with stochastic gradient descent is discussed later) on $U$ and $V$ using the update equations (4.7) and (4.8). ${ }^{4}$ At each iteration, we evaluate the Root Mean Square Error (RMSE) on the validation set, and terminate training

\footnotetext{
${ }^{4}$ While gradient descent in KPMF involves the inverse of $K_{U}$, we actually don't need to invert the matrix when using the CT kernel or the RL kernel since $K_{C T}^{-1}=L$ and $K_{R L}^{-1}=I+\gamma L$.
} 


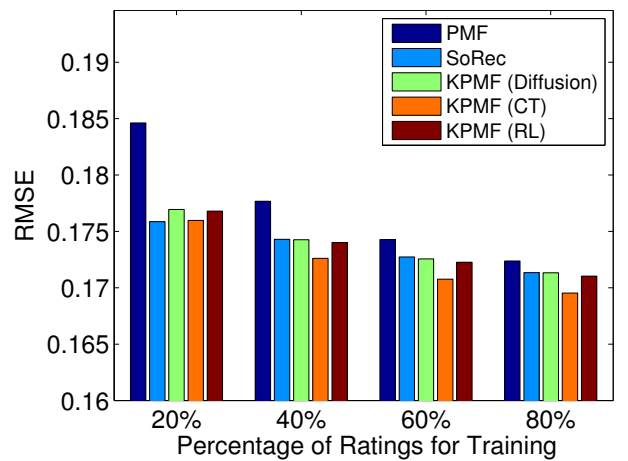

(a) Flixster, $\mathrm{D}=5$

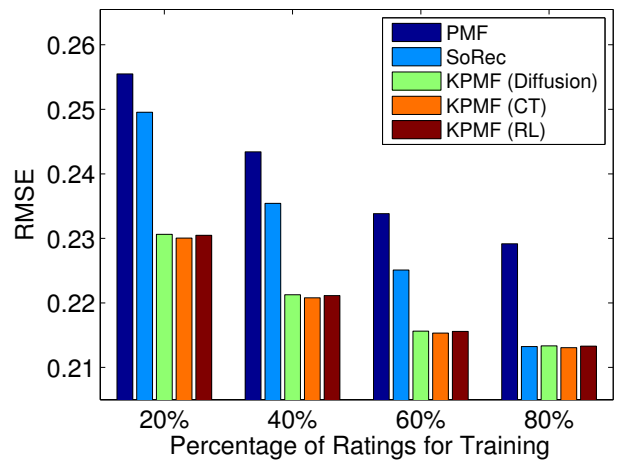

(c) Epinion, $\mathrm{D}=5$

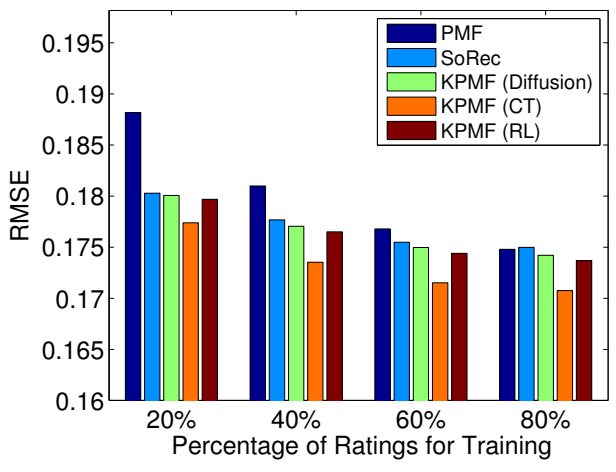

(b) Flixster, $\mathrm{D}=10$

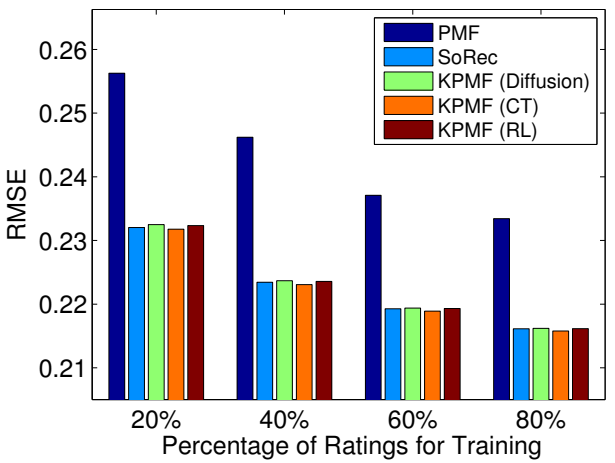

(d) Epinion, $\mathrm{D}=10$

Figure 5: RMSE for different algorithms on Flixster and Epinion datasets (best viewed in color). Lower is better.

once the RMSE starts increasing, or the maximum number of iterations allowed is reached. The learned $U$ and $V$ are then used to predict the ratings in the test set. We run the algorithm with different latent vector dimensions, viz, $D=5$ and $D=10$.

We compare the performance of KPMF (using gradient descent) with three algorithms: The first one uses only the information from the social network. More specifically, to predict the rating $R_{n, m}$, we take the neighbors of user $n$ from the social network, and average their ratings on item $m$ as the prediction for $R_{n, m}$. We denote this method as social network based algorithm (SNB). If none of the neighbors of user $n$ has rated item $m$, SNB cannot predict $R_{n, m}$. The second algorithm we compare with is PMF [1], which only uses the information from the rating matrix. ${ }^{5}$ The third algorithm is SoRec [3], a state-of-the-art collaborative filtering algorithm that combines information from both the rating matrix and the social network by performing matrix factorization jointly. In addition, we also compare the

\footnotetext{
${ }^{5} \mathrm{BPMF}$ actually performs worse than PMF in our experiments, which might be due to improper parameter setting in the code published by the authors. Thus we omit reporting BPMF results here.
}

computational efficiency between KPMF using gradient descent and KPMF using stochastic gradient descent.

Another experiment is prediction for users with no past ratings. We test on 200 users who have most connections in the social network (so that the effect of the social network is most evident). All the past ratings made by these 200 users in the training set are not used for learning. Therefore, the observed rating matrix would contain 200 rows of zeros, and the rest 1,800 rows remain unchanged.

The main measure we use for performance evaluation is Root Mean Square Error (RMSE):

$$
R M S E=\sqrt{\frac{\sum_{i=1}^{n}\left(r_{i}-\hat{r}_{i}\right)^{2}}{n}}
$$

where $r_{i}$ denotes the ground-truth rating value, $\hat{r}_{i}$ denotes its predicted value, and $n$ is the total number of ratings to be predicted.

For SNB, since we cannot do missing value prediction for some entries if none of the corresponding user's neighbors has rated the target item, we also define a measure of coverage for reference, which is defined as the percentage of ratings that can be predicted among all the test entries. 


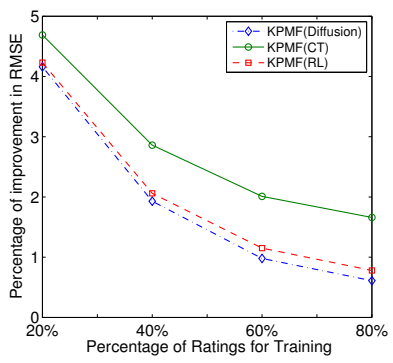

(a) Flixster, $\mathrm{D}=5$

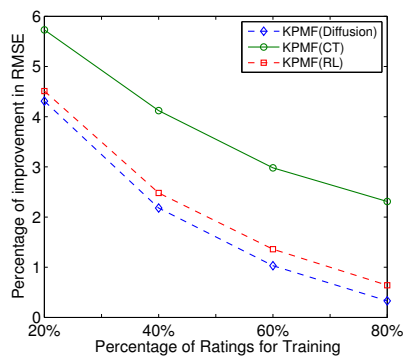

(b) Flixster, $\mathrm{D}=10$

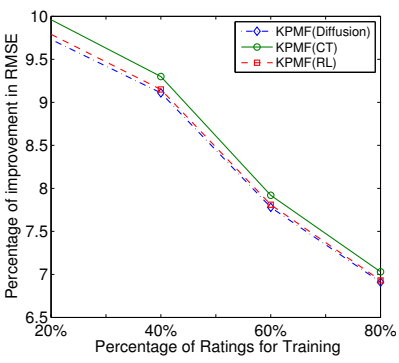

(c) Epinion, $\mathrm{D}=5$

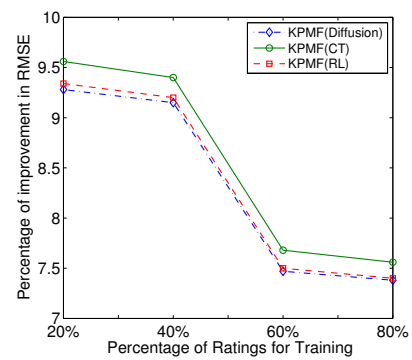

(d) Epinion, $\mathrm{D}=10$

Figure 6: Performance improvement of KPMF compared to PMF on training sets with different number of observed ratings (best viewed in color). The improvement of KPMF versus PMF decreases as more training data are used. This is because for sparser datasets, PMF would have relatively more difficulty in learning users' preferences from fewer number of past ratings, while KPMF could still take advantage of the known social relations among users and utilize the observed ratings better.

(a) Flixster

\begin{tabular}{|l|c|c|c|c|}
\hline Training data used & $20 \%$ & $40 \%$ & $60 \%$ & $80 \%$ \\
\hline RMSE & 0.289 & 0.280 & 0.272 & 0.267 \\
\hline Coverage & 0.31 & 0.50 & 0.62 & 0.70 \\
\hline
\end{tabular}

(b) Epinion

\begin{tabular}{|l|c|c|c|c|}
\hline Training data used & $20 \%$ & $40 \%$ & $60 \%$ & $80 \%$ \\
\hline RMSE & 0.276 & 0.264 & 0.259 & 0.253 \\
\hline Coverage & 0.45 & 0.64 & 0.73 & 0.80 \\
\hline
\end{tabular}

Table 2: RMSE and Coverage from SNB on Flixster and Epinion.

5.4 Results The RMSE on Flixster and Epinion for PMF, SoRec, and KPMF (with different kernels) are given in Figure 5. In each plot, we show the results with different number of ratings used for training, ranging from $20 \%$ to $80 \%$ of the whole dataset. The main observations are as follows:

1. KPMF, as well as SoRec, outperforms PMF on both Flixster and Epinion, regardless of the number of ratings used for training. While KPMF and SoRec use both the social network and the rating matrix for training, PMF uses the rating matrix alone. The performance improvement of KPMF and SoRec over PMF suggests that social network is indeed playing a role in helping predict the ratings. In addition, KPMF also outperforms SoRec for most cases.

2. Figure 6 shows KPMF's percentage of improvement compared to PMF in terms of RMSE. For KPMF, we can see that the performance gain increases as the training data gets sparser. It implies that when the information from the rating matrix is getting weaker, the users' social network is getting more useful for prediction.

3. As shown in Figure 5, among the three graph kernels examined, the CT kernel leads to the lowest RMSE on both Flixster and Epinion. The advantage is more obvious on Flixster than on Epinion.

4. We also give the RMSE of SNB in Table 2 for reference. RMSE for this simple baseline algorithm is much higher than the other algorithms. The coverage is low with a sparse training matrix, but gets higher when the sparsity decreases.

Table 3 shows the results for the experiment of prediction for users with no past ratings. The RMSE are over the selected 200 users who have most connections in the social network, and their past ratings are not utilized during training. To contrast, we only show results on the datasets with $20 \%$ and $80 \%$ training data. KPMF consistently outperforms Item Average ${ }^{6}$ by a large margin if $20 \%$ training data are used, but the advantage is not so obvious for the dataset of $80 \%$ training data (Note that Item Average actually outperforms KPMF on Epinion for Diffusion and RL kernels). This result again implies that the side information from users' social network is more valuable when the observed rating matrix is sparse, and the sparsity is indeed often encountered in real data [2].

Finally, we compare the computational efficiency between KPMF with stochastic gradient descent $\left(\mathrm{KPMF}_{S G D}\right)$ and KPMF with gradient descent $\left(\mathrm{KPMF}_{G D}\right)$. Table 4 shows the RMSE results and running time for the two, where we set $D=10$ and use $p \%=20 \%$ of ratings for training. Although $\mathrm{KPMF}_{S G D}$ has slightly higher RMSE than $\mathrm{KPMF}_{G D}$, it is hundreds of times faster. Similar results are also observed in experiments with other choices of $D$ and $p \%$. Therefore,

\footnotetext{
${ }^{6}$ The algorithm that predicts the missing rating for an item as the average of its observed ratings by other users.
} 
(a) $20 \%$ training data used

\begin{tabular}{|l|c|c|c|c|}
\hline \multirow{2}{*}{} & \multicolumn{2}{|c|}{ Flixster } & \multicolumn{2}{c|}{ Epinion } \\
\cline { 2 - 5 } & $\mathrm{D}=5$ & $\mathrm{D}=10$ & $\mathrm{D}=5$ & $\mathrm{D}=10$ \\
\hline Item Average & 0.2358 & 0.2358 & 0.3197 & 0.3197 \\
\hline KPMF(Diffusion) & 0.2183 & 0.2180 & 0.2424 & 0.2436 \\
\hline KPMF(CT) & 0.2184 & 0.2180 & $\mathbf{0 . 2 3 7 5}$ & $\mathbf{0 . 2 3 7 8}$ \\
\hline KPMF(RL) & $\mathbf{0 . 2 1 8 2}$ & $\mathbf{0 . 2 1 7 9}$ & 0.2422 & 0.2433 \\
\hline
\end{tabular}

(b) $80 \%$ training data used

\begin{tabular}{|l|c|c|c|c|}
\hline \multirow{2}{*}{} & \multicolumn{2}{|c|}{ Flixster } & \multicolumn{2}{c|}{ Epinion } \\
\cline { 2 - 5 } & $\mathrm{D}=5$ & $\mathrm{D}=10$ & $\mathrm{D}=5$ & $\mathrm{D}=10$ \\
\hline Item Average & 0.2256 & 0.2256 & 0.2206 & 0.2206 \\
\hline KPMF(Diffusion) & 0.2207 & 0.2209 & 0.2257 & 0.2269 \\
\hline KPMF(CT) & 0.2209 & 0.2208 & $\mathbf{0 . 2 1 8 0}$ & $\mathbf{0 . 2 1 8 0}$ \\
\hline KPMF(RL) & $\mathbf{0 . 2 2 0 6}$ & $\mathbf{0 . 2 2 0 7}$ & 0.2252 & 0.2263 \\
\hline
\end{tabular}

Table 3: RMSE on users with no ratings for training.

\begin{tabular}{|l|c|c||c|c|}
\hline \multirow{2}{*}{} & \multicolumn{2}{|c||}{ Flixster } & \multicolumn{2}{c|}{ Epinion } \\
\cline { 2 - 5 } & $\mathrm{KPMF}_{G D}$ & $\mathrm{KPMF}_{S G D}$ & $\mathrm{KPMF}_{G D}$ & $\mathrm{KPMF}_{S G D}$ \\
\hline RMSE & $\mathbf{0 . 1 8 0}$ & 0.184 & $\mathbf{0 . 2 3 2}$ & 0.240 \\
\hline Time (sec) & 1353.6 & $\mathbf{5 . 5}$ & 1342.3 & $\mathbf{7 . 8}$ \\
\hline
\end{tabular}

Table 4: Comparison of RMSE and running time for $\mathrm{KPMF}_{G D}$ and $\mathrm{KPMF}_{S G D} . \mathrm{KPMF}_{S G D}$ is slightly worse than $\mathrm{KPMF}_{G D}$ in terms of RMSE, but significantly faster.

for large scale datasets in real applications, $\mathrm{KPMF}_{S G D}$ would be a better choice.

\section{Experiments on Image Restoration}

In this section, we demonstrate the use of KPMF in image restoration to further illustrate the broad potential of this proposed framework and the relevance of incorporating side information. Image restoration is the process of recovering corrupted regions of a target image [5]. Let us denote the $N \times M$ target image by $P$, and the corrupted region to be recovered by $\Omega$ (see black scribbles on the second column of Figure 8 ). The task is to fill in the pixels inside $\Omega$ in a way that is coherent with the known pixels outside $\Omega$, i.e. $P-\Omega$. Now one might notice that this problem is quite similar to the one faced in recommender systems, where the rating matrix $R$ becomes $P$, ratings become pixel values, and the missing entries become $\Omega$. Therefore, if we consider the rows of the image as users and the columns as items, we could apply the KPMF algorithm to fill in the pixels in $\Omega$ just as predicting missing entries in recommender systems. However, since no direct information of correlations among rows or columns of the image is given, the difficulty arises in obtaining proper kernel matrices.

One way to address this is to construct such a graph for images in analogy to the users' social network for recommender systems. To do that, we consider the spatial smoothness in an image (while this will be used here to illustrate the proposed framework, we can consider graphs derived from other attributes as well, e.g., smoothness in feature space, with features derived from local patches or texture-type multiscale analysis [19]). Below we describe how to construct

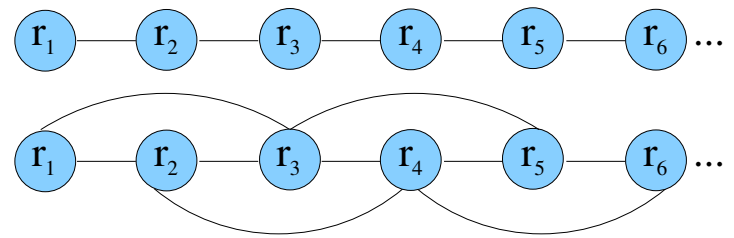

Figure 7: The graph constructed for the rows of the image using the spatial smoothness property. (Top: $\Delta=1$, Bottom: $\Delta=2$.)

the graph for rows using this property (the graph for columns can be constructed in a similar fashion). First, we assume that each row is similar to its neighboring rows and thus directly connected in the graph. Let $r_{i}$ $(i=1, \ldots, N)$ be the node in the graph that represents the $i^{\text {th }}$ row of the image (nodes representing parts of the image rows could be considered as well to further localize the structure). Then there exists an edge between $r_{i}$ and $r_{j}(j \neq i)$ if and only if $|i-j| \leq \Delta$, where $\Delta$ is a constant that determines the degree of $r_{i}$ (see Figure 7). The corresponding adjacency matrix of the graph is a band matrix with 1's confined to the diagonal band and 0's elsewhere.

Given the graphs for rows and columns, we can obtain their corresponding kernel matrices by applying the graph kernels from Section 5.2. Since each color image is composed of three channels (Red, Green and Blue), the KPMF update equations for learning the latent matrices are applied to each channel independently. Finally, the estimation for $\Omega$ from the three channels, along with the known pixels in $P-\Omega$, are combined together to form the restored image. Restoration results using PMF and KPMF on several corrupted images are shown in Figure 8 , and the RMSE comparison is given in Table 5 (note that all pixel values are normalized to $[0,1]$.). Unlike KPMF that is able to utilize the spatial smoothness assumption, PMF can only use the observed pixels in the image for restoration. Thus, as expected, its restoration quality is worse than KPMF. 


\begin{tabular}{|l|c|c|c|c|c|c|c|}
\hline & \multicolumn{3}{|c|}{ KPMF } & \multicolumn{3}{c|}{ PMF } & \\
\hline Image & Channel R & Channel G & Channel B & Channel R & Channel G & Channel B & \#Masked pixels \\
\hline 1 & 0.082 & 0.073 & 0.066 & 0.120 & 0.101 & 0.094 & 5426 \\
\hline 2 & 0.160 & 0.164 & 0.156 & 0.173 & 0.177 & 0.170 & 5975 \\
\hline 3 & 0.135 & 0.133 & 0.131 & 0.179 & 0.171 & 0.164 & 31902 \\
\hline 4 & 0.100 & 0.106 & 0.124 & 0.149 & 0.141 & 0.173 & 32407 \\
\hline 5 & 0.103 & 0.049 & 0.030 & 0.143 & 0.081 & 0.040 & 28025 \\
\hline 6 & 0.109 & 0.091 & 0.081 & 0.141 & 0.109 & 0.105 & 23061 \\
\hline
\end{tabular}

Table 5: RMSE comparison between PMF and KPMF on RGB channels of restored images. Smaller is better.

\section{Conclusion}

We have presented a new matrix completion algorithm, i.e. KPMF, which exploits the underlying correlations among rows and among columns of the data matrix simultaneously for missing value prediction. KPMF introduces Gaussian process priors for latent matrices in the generative model, which forces the learned latent matrices to respect the covariance structure among rows and among columns, enabling the incorporation of side information when learning the model. As demonstrated in the experiments, this characteristic could play a critical role in boosting the model performance, especially when the observed data matrix is sparse.

Another advantage of KPMF over PMF and BPMF is its ability to predict even when an entire row/column of the data matrix is missing as long as appropriate side information is available. In principle, KPMF is applicable to general matrix completion problems, but in this paper we focus on two specific applications: recommender systems and image restoration. In the future, we would like to generalize the current model to handle the case of weighted entries, where different entries are assigned different weights according to some pre-defined criteria (e.g. for item recommendation, we may want the model to place more emphasis on infrequent users/items during training).

\section{Acknowledgments}

The research was supported by NSF grants IIS-0812183, IIS-0916750, IIS-1029711, and NSF CAREER award IIS-0953274. Guillermo Sapiro is supported by NSF, ONR, ARO, NGA, NSSEFF, and DARPA.

\section{References}

[1] R. Salakhutdinov and A. Mnih, "Probabilistic matrix factorization," in NIPS, 2007.

[2] B. M. Sarwar, G. Karypis, J. A. Konstan, and J. Reidl, "Item-based collaborative filtering recommendation algorithms," in $W W W$, ser. 10, 2001, pp. 285-295.

[3] H. Ma, H. Yang, and I. K. M. Lyu, "Social recommendation using probabilistic matrix factorization," in CIKM, 2008.
[4] H. Wackernagel, Multivariate Geostatistics: An Introduction With Applications. Springer-Verlag, 2003.

[5] M. Bertalmio, G. Sapiro, V. Caselles, and C. Ballester, "Image inpainting," in Proceedings of ACM SIGGRAPH, 2000, pp. 417-424.

[6] N. Lawrence and R. Urtasun, "Non-linear matrix factorization with Gaussian processes," in ICML, 2009.

[7] R. Salakhutdinov and A. Mnih, "Bayesian probabilistic matrix factorization using Markov chain Monte Carlo," in $I C M L, 2008$.

[8] H. Shan and A. Banerjee, "Generalized probabilistic matrix factorizations for collaborative filtering," in ICDM, 2010.

[9] A. Agovic, A. Banerjee, and S. Chatterjee, "Probabilistic matrix addition," in $I C M L, 2011$.

[10] C. E. Rasmussen, Gaussian Processes in Machine Learning. Springer, 2004.

[11] M. Jamali and M. Ester, "A matrix factorization technique with trust propagation for recommendation in social networks," in RecSys, 2010.

[12] P. Massa and P. Avesani, "Trust metrics in recommender systems," in Computing with Social Trust. Springer London, 2009.

[13] F. Chung, Spectral graph theory. American Mathematical Society, 1997.

[14] B. Schölkopf and A. Smola, Learning with Kernels. MIT Press, 2001.

[15] R. Kondor and J. Lafferty, "Diffusion kernels on graphs and other discrete structures," in ICML, 2001.

[16] F. Fouss, A. Pirotte, J.-M. Renders, and M. Saerens, "Random-walk computation of similarities between nodes of a graph with application to collaborative recommendation," IEEE Transactions on Knowledge and Data Engineering, vol. 19, no. 3, pp. 355-369, 2007.

[17] L. Lovasz, "Random walks on graphs: a survey," Combinatorics, Paul Erdos is Eighty, vol. 2, pp. 353397, 1996.

[18] A. Smola and R. Kondor, "Kernels and regularization on graphs," The Sixteenth Annual Conference on Learning Theory/The Seventh Workshop on Kernel Machines, 2003.

[19] A. Efros and T. Leung, "Texture synthesis by nonparametric sampling," in Proc. ICCV, 1999, pp. 10331038. 

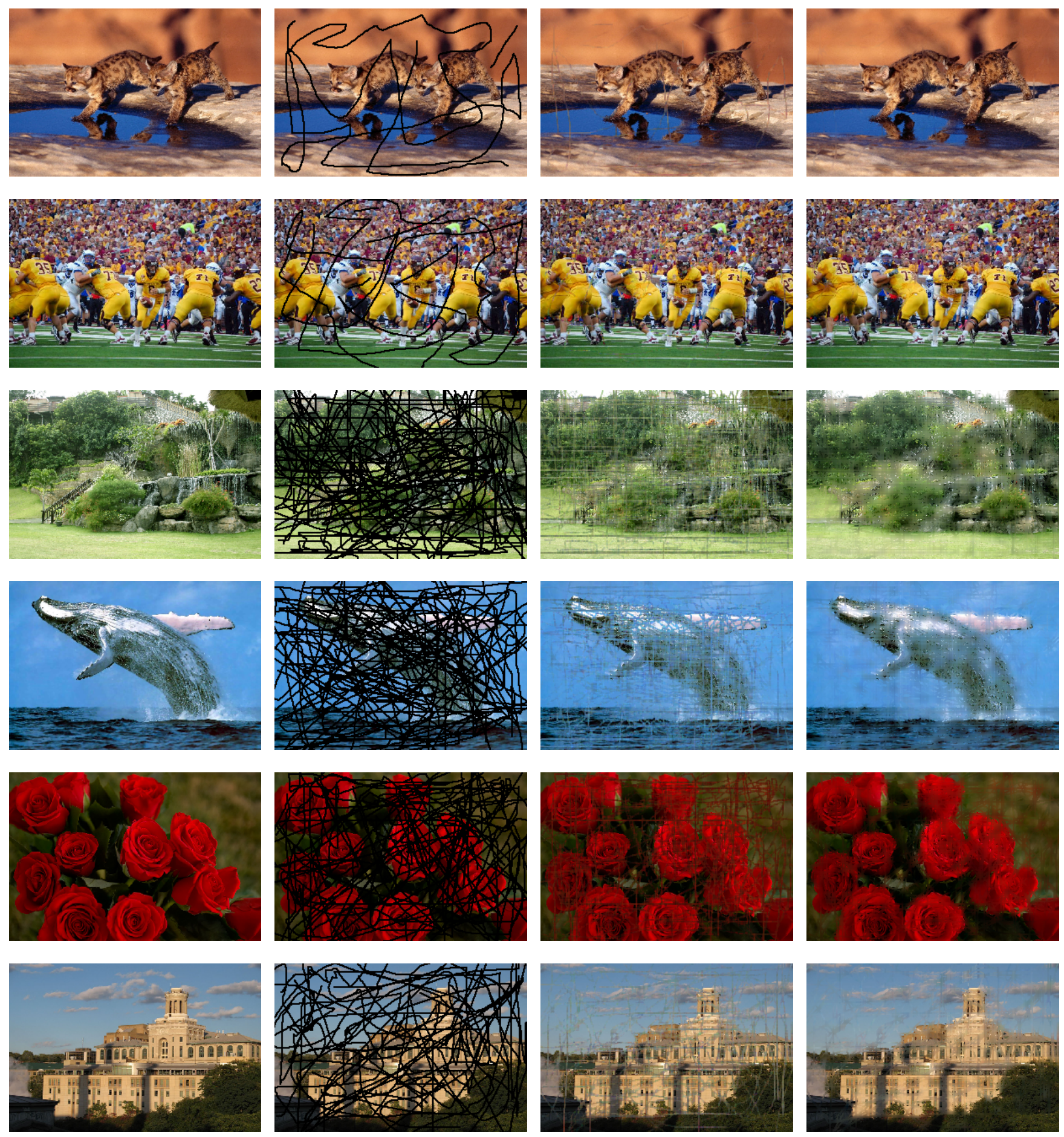

Figure 8: Image restoration results using PMF and KPMF (best viewed in color). From left to right: original images, corrupted images (regions to be restored are in black), images restored using PMF, and images restored using KPMF. For KPMF, $\Delta$ equals to 5 when constructing the row and column graphs, and Diffusion kernel with $\beta=0.5$ is used to obtain the kernel matrices. 